\title{
Declaración de Cartagena desde la óptica de la presidencia de la FELANPE
}

\author{
Declaration of Cartagena from the optics of the presidency of FELANPE \\ Declaração de Cartagena da óptica da presidência de FELANPE
}

\author{
Dolores Rodríguez Veintimilla, MD. ${ }^{1}$
}

https://doi.org/10.35454/rncm.v2supl1.027

De acuerdo con la Real Academia Española (RAE), Declaración es la acción y efecto de declarar, manifestación o explicación de lo que los otros dudan o ignoran, manifestación del ánimo o la intención.

Con fecha 3 de mayo del 2019, los 16 países que integran la Federación Latinoamericana de Terapia Nutricional, Nutrición Clínica y Metabolismo (FELANPE) en la ciudad de Cartagena, Colombia, alzaron su voz, proclamaron al mundo y registraron su real compromiso de reconocer el cuidado nutricional como un Derecho Humano independiente del nivel de atención en salud, en acción contra las diversas clases de malnutrición y en particular la que está asociada a la enfermedad, convencidos de que una adecuada Terapia Nutricional puede corregir la malnutrición, mejorar el pronóstico de la enfermedad, la calidad de vida, disminuir las comorbilidades, la mortalidad y los costos en salud.

La gran iniciativa de reconocer el derecho de los pacientes a recibir terapia nutricional como un derecho humano, registrada como Declaración de Cartagena: declaración internacional sobre el derecho al cuidado nutricional y la lucha contra la malnutrición, vio la luz de la mano de una gran profesional, la doctora Diana Cárdenas quien con el doctor Charles Bermúdez, presidente, y otros distinguidos miembros de la Asociación Colombiana de Nutrición Clínica (ACNC) diseñaron este gran proyecto e impulsaron esta actividad con el objetivo principal de disminuir la prevalencia de malnu-

1 Presidente FELANPE 2019 - 2020

*Correspondencia: Dolores Rodríguez Veintimilla dra.rodriguezv@yahoo.com trición asociada a la enfermedad y favorecer el desarrollo de la nutricion clinica. Las cifras de malnutrición oscilan en América Latina entre $40 \%$ y $60 \%$, encontrándose, incluso, estudios que reportan incremento en su prevalencia con notable aumento de los días de estancia hospitalaria y mayor frecuencia de comorbilidades.

La pregunta viene a la mente: ¿Cómo impulsar el cumplimiento de la Declaración de Cartagena? La respuesta se concreta en tres palabras: Conocimiento, Decisión y Perseverancia, enmarcadas en una respuesta coordinada y con directrices claras en el trabajo a realizarse, pues su implementación podría contribuir con los Objetivos del Desarrollo Sostenible de las Naciones Unidas, lo que nos conduce al último punto de la Declaración en el cual la FELANPE comparte con sus organizaciones corresponsables la preocupación y la ocupación en cuanto al proceso de intervenir las distintas formas de la malnutrición, como consecuencia del impacto negativo que tiene sobre la salud de las personas, comunidades y sociedades.

Es gratificante observar la forma como se está desarrollando el trabajo conjunto de los diferentes países de FELANPE para luchar contra la malnutrición hospitalaria, y este ejemplo de perseverancia y esfuerzo ha trascendido a otras sociedades como la Sociedad Europea de Nutrición Clínica y Metabolismo (ESPEN), la Sociedad Americana de Nutrición Parenteral y Enteral (ASPEN), y la Federación Panamericana e Ibérica de Medicina Crítica y Terapia Intensiva (FEPIMCTI), entre otras.

Como se ha expresado en múltiples ocasiones "ninguna persona es una isla", y los objetivos deseados no se lograrán si cada uno trabaja solo, por su lado. En tal sentido, con la firma y proclamación de la Declaración de Cartagena, la FELANPE lidera un esfuerzo global 
para que finalmente la seguridad alimentaria y nutricional en los hospitales y sistemas de salud sea reconocida como parte indisoluble del éxito terapéutico y la gestión sanitaria integral.

\section{Referencias bibliográficas}

1. Cardenas D, Bermúdez CH, Echeverri S, Perez A, Puentes M, Lopez M, et al. Declaración de Cartagena. Declaración
Internacional sobre el Derecho al Cuidado Nutricional y la Lucha contra la Malnutrición. Nutr Hosp. 2019;36(4):97498. http://dx.doi.org/10.20960/nh.02701.

2. Correia MITD, Perman MI, Waitzberg DL. Hospital malnutrition in Latin America: a systematic review. Clin Nutr. 2017;36:958-67.

3. Ruiz AJ, Buitrago G, Rodríguez N, Gómez G, Sulo S, Gómez $\mathrm{C}$, et al. Clinical and economic outcomes associated with malnutrition in hospitalized patients. Clin Nutr. 2018; S02615614(18)30201-2. doi: 10.1016/j.clnu.2018.05.016. 